\title{
Two CHRN susceptibility variants for COPD are genetic determinants of emphysema and chest computed tomography manifestations in Chinese patients
}

\author{
This article was published in the following Dove Press journal: \\ International Journal of COPD \\ 15 May 2017 \\ Number of times this article has been viewed
}

\section{Zhuxiang Zhao',2 \\ Changbin Jiang' \\ Dongxing Zhao' \\ Yujun $\mathrm{Li}^{2}$ \\ Chunxiao Liang ${ }^{\prime}$ \\ Weifeng Liu ${ }^{2}$ \\ Shuquan $\mathrm{Wei}^{2}$ \\ Yumin Zhou' \\ Ziwen Zhao ${ }^{2}$ \\ Pixin Ran'}

'The State Key Laboratory of Respiratory Diseases, The First Affiliated Hospital, Guangzhou Medical University, Guangzhou, ${ }^{2}$ The Pulmonary Medicine, Guangzhou First People's Hospital, Guangzhou Medical University, Guangzhou, Guangdong,

People's Republic of China
Correspondence: Pixin Ran The State Key Laboratory of Respiratory Diseases, The First Affiliated Hospital, Guangzhou Medical University, I5I Yanjiang Rd., Guangzhou, Guangdong 5I012, People's Republic of China Email pxran@gzhmc.edu.cn

Ziwen Zhao

The Pulmonary Medicine, Guangzhou First People's Hospital, Guangzhou Medical University, No 602 renminbei Rd., Guangzhou, Guangdong 510182 , People's Republic of China Email zhaozw@yeah.net

\begin{abstract}
Quantitative computed tomography (CT) measures of emphysema have been shown to be associated with increased mortality in humans, but genetic variants affecting the quantitative parameters of chest $\mathrm{CT}$ that measure degree of emphysema have not yet been examined. In this study, using available chest CT data from a total of 344 emphysema patients, we assessed the correlations between five chronic obstructive pulmonary disease (COPD) susceptibility variants in the cholinergic receptor nicotinic $(C H R N)$ genes and the degree of emphysema and chest CT manifestations. We verified that most of the parameters were significantly correlated with the degree of emphysema. Compared to rs76071148AA and TT genotype carriers, the rs76071148AT genotype carriers exhibited a decreased probability of having severe emphysema (odds ratio $[\mathrm{OR}]=0.63,95 \%$ confidence interval $[\mathrm{CI}]=0.40-0.99$ ), whereas the variant rs $8040868 \mathrm{C}$ allele was negatively correlated with the emphysema index $(P=0.002)$. Interestingly, further stratification analysis grouped by spirometry-diagnosed COPD status revealed that the variant $\mathrm{rs} 8040868 \mathrm{C}(\mathrm{CT}+\mathrm{CC})$ genotypes exerted a protective effect against severe emphysema with borderline significance ( $\mathrm{OR}=0.41,95 \% \mathrm{CI}=0.16-1.05)$ and affected the mean lung density, emphysema index, ratio of airway wall thickness to airway dimensions (AWT/AD), and AWT grade in spirometry-diagnosed non-COPD subjects. The rs76071148 variant was also significantly associated with AWT/AD and AWT grade in those individuals. In summary, we determined that rs 8040868 and rs 76071148 are promising indicators of the degree of emphysema and chest CT manifestations, especially in spirometry-diagnosed non-COPD subjects.
\end{abstract}

Keywords: COPD, CHRN variant, CT manifestation, emphysema

\section{Introduction}

Chronic obstructive pulmonary disease (COPD) is a common public health problem characterized by persistent airflow limitation that is usually progressive. Part of the airflow limitation is directly attributed to emphysema. ${ }^{1}$ Emphysema is characterized by the destruction of the lung parenchyma by inflammatory processes, oxidative stress, and apoptosis, which can lead to the loss of alveolar attachments to the small airways and decreased lung elastic recoil. Normally, airflow limitation is measured by spirometry. However, spirometry fails to discriminate bronchial obstruction in a small number of patients with emphysema. Lutchmedial et al reported that spirometry failed to detect $0.4 \%$ of patients with clinical COPD who exhibited significant emphysema on chest computed tomography (CT) scans. ${ }^{2}$ Chest CT offers an anatomic picture of COPD, 
enabling the discrimination of the state of emphysema. Moreover, CT-measured emphysema is a strong independent predictor of mortality in humans. ${ }^{3}$

Differential susceptibility to COPD development is suggested by the fact that siblings of patients with severe COPD exhibit a significant risk of airflow obstruction. ${ }^{4}$ This differential susceptibility has been suggested to be due in part to genetic variants in genes that are correlated with the pathogenesis of COPD..$^{5-8}$ The cholinergic receptor nicotinic $(C H R N)$ genes, including alpha 3 (CHRNA3), beta 4 (CHRNB4), and alpha 5 (CHRNA5), are located on $15 \mathrm{q} 25$ and are the most recognized susceptibility genes for COPD. ${ }^{9}$ The genetic variants of the $C H R N$ genes are well-established to be associated with COPD and lung function traits by spirometry in multiple ethnicities across different studies. ${ }^{10-12}$ In our previous studies, we also identified an exon variant in CHRNA3-CHRNB4-CHRNA5 that was significantly associated with COPD risk in Chinese patients. ${ }^{13,14}$ However, few studies have investigated the effect of these variants on CTmeasured emphysema in Chinese populations.

CT-measured emphysema is associated with severe lung function impairment. Moreover, the presence of emphysema is correlated with a low body mass index (BMI), reduced diffusion capacity and an increased risk of lung cancer. To date, only studies conducted in Western countries have shown that these $C H R N$ variants are associated with emphysema. ${ }^{15-18}$ Thus, validating the association in other ethnicities is paramount. Moreover, the effects of these $C H R N$ variants on CT-measured parameters remain unknown.

In this study, using available quantitative CT data from a total of 344 emphysema patients, we aimed to investigate the associations between five genetic variants of the $C H R N$ genes and the degree of emphysema. We also analyzed the effects of these variants and their interactions with smoking on a series of chest CT manifestations, including mean lung density (MLD), percentage of low attenuation area volume (LAA\%), emphysema volume (EV), emphysema index (EI), airway wall thickness (AWT), the ratio of airway wall thickness with airway dimensions (AWT/AD), and AWT grade. The associations were further subjected to stratification analysis with regard to the spirometry-diagnosed COPD condition.

\section{Materials and methods Study subjects}

In this cross-sectional study, we randomly recruited 344 emphysema participants from the First Affiliated Hospital of Guangzhou Medical University and Guangzhou First People's Hospital, Guangzhou Medical University between
February 2012 and September 2013. Each patient was asked to donate a $5-\mathrm{mL}$ blood sample and was interviewed with a questionnaire to collect data on sex and smoking status. All measurements of pulmonary function were performed using portable spirometers (Micro Medical Ltd, Chatham, Kent, UK) according to the guidelines of the American Thoracic Society. ${ }^{19}$ The predicted forced expiratory volume in $1 \mathrm{~s}$ $\left(\mathrm{FEV}_{1}\right)$ was based on the reference value of the European Coal and Steel Community with adjustment for the Chinese population (males 0.95 and females 0.93 ). The presence of bronchial obstruction was defined by a post-bronchodilator $\mathrm{FEV}_{1} /$ forced vital capacity $(\mathrm{FVC})$ ratio $<0.70$. All participants signed written informed consent forms prior to participation in the study. We failed to collect data on pack-years because a significant proportion of the studied sample could not accurately quantify previous tobacco use. The study was approved by the medical ethics committee of the Guangzhou Institute of Respiratory Diseases and the medical ethics committee of the Guangzhou First People's Hospital, Guangzhou Medical University on 2 November 2013.

\section{Measurement of emphysema}

In the cross-sectional study, the subjects underwent a chest high-resolution CT scan by a 320-slice spiral CT scanner (Toshiba Medical Systems, Otawara, Japan) at full inspiration. Two blinded radiologists independently reviewed each CT scan. The extent of radiographic emphysema was quantitatively assessed by a computer-aided diagnosis (Newsoft Medical Systems Co., Ltd., Shengyang, China). In addition, the chest CT manifestations were scored, including the MLD, $\mathrm{EV}$, and EI. The extent of emphysema was estimated using the LAA\% (the percent of voxels with an apparent X-ray attenuation value below -950 HU). Emphysema was reported as mild, moderate, or severe if the LAA $\%$ was $5 \%-25 \%$, $25 \%-50 \%$, or $>50 \%$ of the lung, respectively. Thickening of the bronchial airway walls was quantitatively scored by Apollo Pulmonary Evaluation Software (ver2.0, VIDA Diagnostics, Coralville, IA, USA). The extent of airway wall thickening was quantitatively measured in the fourth generations of the apical segment of the right upper lobe. ${ }^{20}$ The airway external diameter (D) and lumen diameter (L) were measured. The AWT was defined as follows: AWT = (D-L)/2. The AWT/AD and AWT grade were analyzed.

\section{Genotyping}

Genomic DNA was extracted from the blood samples. The sample's genotyping and quality control procedures have been described previously. ${ }^{13,14}$ Briefly, five single 
nucleotide polymorphisms (SNPs) (rs1051730C $>\mathrm{T}$ and rs8040868A $>\mathrm{G}$ of $C H R N A 3$, rs $56218866 \mathrm{~A}>\mathrm{G}$ of $C H R N B 4$ and $\mathrm{rs} 16969968 \mathrm{G}>\mathrm{A}$ and $\mathrm{rs} 76071148 \mathrm{~T}>\mathrm{A}$ of $C H R N A 5)$ were selected as described in our previous study. The samples were genotyped by high-resolution melt curve analysis and direct sequencing.

\section{Statistical analysis}

Differences in the characteristics, chest CT parameters and pulmonary function between groups stratified by the degree of emphysema and spirometry-diagnosed COPD status were assessed using one-way analysis of variance for measurement data and the $\chi^{2}$ test for enumeration data. Associations between the $C H R N$ variants and emphysema severity were tested using a logistic regression model with age, sex, smoking status, and BMI as covariables. We used the Spearman's Rank Correlation test to assess associations between these 5 SNPs. Chest CT manifestations were analyzed as continuous variables. The generalized linear model (GLM) was used to evaluate the correlations between $C H R N$ variants and chest CT manifestations with variants as predictors, CT data as outcomes, and age, sex, BMI, smoking status, and degree of emphysema as adjusted variables based on the additive model. The possible "gene-gene" or "geneenvironment" interactions were assessed using generalized multifactor dimensionality reduction (GMDR) software. ${ }^{21}$ SAS software (version 9.4; SAS Institute, Cary, NC, USA) was applied to perform the above analyses, and a $P$-value of $<0.05$ was considered statistically significant.

\section{Results}

The selected characteristics and chest CT parameters stratified by the degree of emphysema are presented in Table 1. The factors sex, age, smoking status, spirometry-diagnosed COPD, post-FVC, post-FEV $/$ FVC, MLD, EV, LAA\%, EI, AWT, and AWT grade, but not post-FEV 1 and AWT/AD, differed significantly between subjects with different degrees of emphysema, which is consistent with the clinical features driven by emphysema. Moreover, it is worth noting that the spirometry test is more inclined to fail in discriminating COPD patients with severe emphysema from those with mild or moderate emphysema $(P<0.001)$. In addition, there were some non-smoking patients, suggesting the presence of other exposures causing emphysema in Chinese patients, such as air pollution.

We first assessed the associations between $C H R N$ variants and the degree of emphysema in all subjects. As shown in Table 2, although no significant differences were observed in the distribution of phenotype frequency for each variant between subjects with different degrees of emphysema, we detected an association approaching statistical significance between the rs76071148AT genotype and the risk of emphysema with an odds ratio (OR) of 0.66 (95\% confidence interval $[\mathrm{CI}]=0.68-1.06 ; P=0.084)$. We then applied all possible genetic models for associations between each variant and the degree of emphysema. Because Akaike's information criterion value was the smallest, the rs76071148 variant best fit the heterozygous model, in which AT genotype carriers exhibited a lower probability of having severe emphysema

Table I Distributions of the select characteristics and chest CT parameters with regard to degree of emphysema

\begin{tabular}{|c|c|c|c|c|}
\hline \multirow[t]{2}{*}{ Variables } & \multicolumn{3}{|c|}{ Degree of emphysema $^{a}$} & \multirow[t]{2}{*}{$P$-value ${ }^{\mathrm{b}}$} \\
\hline & Mild (n) & Moderate (n) & Severe $(n)$ & \\
\hline Total no & 68 & 86 & 190 & \\
\hline Sex & $M / F(66 / 2)$ & $M / F(74 / I 2)$ & $M / F(131 / 59)$ & $<0.001$ \\
\hline Age (years) & $65.10 \pm 7.67(68)$ & $68.24 \pm 8.93(86)$ & $71.97 \pm 10.30(190)$ & $<0.001$ \\
\hline BMI & $21.36 \pm 3.16(68)$ & $21.30 \pm 3.55(86)$ & $22.89 \pm 3.76(190)$ & $<0.001$ \\
\hline Smoke & $\mathrm{Y} / \mathrm{N}(64 / 4)$ & $\mathrm{Y} / \mathrm{N}(8 \mathrm{I} / 5)$ & Y/N (|4|/49) & $<0.001$ \\
\hline Spirometry-diagnosed COPD & $\mathrm{Y} / \mathrm{N}(58 / 10)$ & Y/N (70/16) & Y/N (II3/77) & $<0.001$ \\
\hline Post-FEV & $1.65 \pm 0.75(68)$ & $1.50 \pm 0.78(86)$ & $1.42 \pm 0.7 \mid(190)$ & 0.091 \\
\hline Post-FVC & $2.94 \pm 0.81(68)$ & $2.55 \pm 0.93(86)$ & $2.15 \pm 0.86(190)$ & $<0.001$ \\
\hline Post-FEV/FVC & $0.54 \pm 0.15(68)$ & $0.57 \pm 0.16(86)$ & $0.65 \pm 0.14(190)$ & $<0.001$ \\
\hline MLD & $0.08 \pm 0.02(68)$ & $0.12 \pm 0.02(86)$ & $0.2 I \pm 0.05(I 88)$ & $<0.001$ \\
\hline LAA $\%$ & $64.77 \pm 9.30(68)$ & $36.54 \pm 6.66(86)$ & $7.020 \pm 8.03(189)$ & $<0.001$ \\
\hline EV & $3,710.93 \pm 891.97(68)$ & $\mathrm{I}, 787.5 \mathrm{I} \pm 502.98(86)$ & $291.91 \pm 361.51(154)$ & $<0.001$ \\
\hline El & $5.84 \pm 0.55(68)$ & $7.36 \pm 0.60(86)$ & $3.17 \pm 3.21(189)$ & $<0.001$ \\
\hline AWT & $1.42 \pm 0.45(59)$ & $1.28 \pm 0.50(74)$ & $1.20 \pm 0.48(168)$ & 0.009 \\
\hline AWT/AD & $37.13 \pm 7.36(57)$ & $35.28 \pm 9.33(69)$ & $33.21 \pm 13.66(162)$ & 0.077 \\
\hline Grading of AWT & 0/1/2 (7/48/2) & $0 / 1 / 2(18 / 48 / 3)$ & $0 / 1 / 2(65 / 87 / / 0)$ & 0.001 \\
\hline
\end{tabular}

Notes: ${ }^{\text {a }} \mathrm{A}$ small portion of data was missing for certain parameters. ${ }^{\mathrm{b}}$ The one-way ANOVA test and $\chi^{2}$ test were used to assess the $P$-value. Data presented as mean \pm standard deviation unless otherwise specified.

Abbreviations: AD, airway dimension; ANOVA, analysis of variance; AWT, airway wall thickness; BMI, body mass index; COPD, chronic obstructive pulmonary disease;

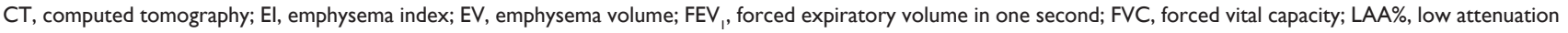
areas volume percentage; M/F, male/female; MLD, mean lung density; Y/N, yes/no. 
Table 2 Distributions of CHRN variants and their associations with degree of emphysema

\begin{tabular}{|c|c|c|c|c|c|}
\hline \multirow[t]{2}{*}{ Variants } & \multicolumn{3}{|c|}{ Degree of emphysema } & \multirow[t]{2}{*}{$P$-value ${ }^{a}$} & \multirow[t]{2}{*}{ OR $(95 \% \mathrm{Cl})^{\mathrm{b}}$} \\
\hline & Mild n (\%) & Moderate n (\%) & Severe n (\%) & & \\
\hline Total subjects & 68 & 86 & 190 & & \\
\hline rs7607|I48 & & & & 0.199 & \\
\hline TT & $38(55.9)$ & $53(61.6)$ & IOI (53.2) & & $\mathrm{I} .00$ (Ref) \\
\hline AT & $23(33.8)$ & $29(33.7)$ & $81(42.6)$ & & $0.66(0.42-1.06)$ \\
\hline $\mathrm{AA}$ & $7(10.3)$ & $4(4.7)$ & $8(4.2)$ & & $1.74(0.68-4.47)$ \\
\hline AT vs TT $+\mathrm{AA}$ & & & & & $0.63(0.40-0.99)$ \\
\hline rs8040868 & & & & 0.393 & \\
\hline $\mathrm{TT}$ & $30(19.1)$ & $36(41.9)$ & $74(39.0)$ & & $\mathrm{I} .00$ (Ref) \\
\hline $\mathrm{CT}$ & $25(36.8)$ & $40(46.5)$ & $93(48.9)$ & & $0.76(0.47-1.22)$ \\
\hline $\mathrm{CC}$ & $13(19.1)$ & $10(11.6)$ & $23(12.1)$ & & $1.14(0.58-2.25)$ \\
\hline rs56218866 & & & & 0.312 & \\
\hline TT & $53(77.9)$ & $63(73.3)$ & I5I (79.5) & & $\mathrm{I} .00$ (Ref) \\
\hline $\mathrm{CT}$ & $14(20.6)$ & $21(24.4)$ & $39(20.5)$ & & $1.13(0.67-1.91)$ \\
\hline $\mathrm{CC}$ & $\mathrm{I}(\mathrm{I} .5)$ & $2(2.3)$ & $0(0.0)$ & & $3.15(0.37-26.8)$ \\
\hline rs16969968/rs 1051730 & & & & 0.336 & \\
\hline GG & $65(95.6)$ & 77 (89.5) & 177 (93.2) & & $\mathrm{I} .00$ (Ref) \\
\hline AG & $3(4.4)$ & $9(10.5)$ & $13(6.8)$ & & $0.94(0.4 I-2.14)$ \\
\hline
\end{tabular}

Notes: ${ }^{\mathrm{a} T h e}$ two-sided $\chi^{2}$ test between difference groups. ${ }^{\mathrm{b}} \mathrm{Adjusted}$ in a logistic regression model with age, sex, smoking status, and BMI as covariables. Abbreviations: $\mathrm{BMI}$, body mass index; $\mathrm{Cl}$, confidence interval; Ref, reference.

than the combination of the rs76071148AA and TT genotype carriers $(\mathrm{OR}=0.63,95 \% \mathrm{CI}=0.40-0.99 ; P=0.047)$, which exerted a heterozygous advantage. However, no other association was observed under any assumed models for each variant (data not shown). In a further stratification analysis grouped by spirometry-diagnosed COPD status as shown in Table S1, we observed that in spirometry-diagnosed non-COPD subjects, the rs8040868C (CT + CC) genotypes conferred a protective effect against severe emphysema under a dominant genetic model $(\mathrm{OR}=0.41 ; 95 \% \mathrm{CI}=0.16-1.05$; $P=0.063$ ). In addition, although a significant difference was detected in the frequency distribution of the two completely linked variants rs16969968 and rs1051730 between individuals with different degrees of emphysema $(P=0.049)$, no significant association was observed between the genotype and emphysema risk in spirometry-diagnosed non-COPD subjects. Furthermore, no other significant correlation was observed between these variants (data not shown).

We further tested the quantitative parameters of chest $\mathrm{CT}$ and CHRN genotype correlations in all subjects and those stratified by spirometry-diagnosed COPD status using the GLM test. As shown in Table 3, only the rs8040868 variant was significantly associated with the EI in all subjects $(P=0.002)$. The EI gradually decreased with the number of rs8040868C alleles (TT: 5.09 $\pm 2.90, \mathrm{CT}$ :

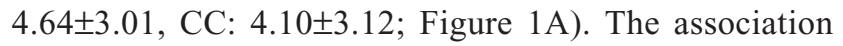
remained significant when stratified by COPD status (in COPD: $P=0.042$; in controls: $P=0.008$; Figure $1 \mathrm{~B}$ and $\mathrm{C}$ ). However, no other significant association was observed for these variants and other CT parameters for all subjects. More interestingly, several significant associations were found in spirometry-diagnosed non-COPD subjects that were not observed in spirometry-diagnosed COPD patients. The rs8040868 variant seemed to be a common genetic determinant of CT parameters with significant effects on the MLD (TT: $0.17 \pm 0.62, \mathrm{CT}: 0.20 \pm 0.08, \mathrm{CC}$ : $0.20 \pm 0.07, P=0.029$; Figure 1D), EI (TT: 4.19 \pm 3.54 , CT: 2.49 $\pm 3.18, \mathrm{CC}: 2.66 \pm 3.32 ; P=0.008)$, and AWT/AD (TT: 31.74 $\pm 11.58, \mathrm{CT}: 38.90 \pm 9.35, \mathrm{CC}: 36.42 \pm 11.58$, $P=0.004$; Figure 1E). The rs76071148 variant was significantly associated with the AWT/AD (TT: 33.99 12.85 , AT: 34.64 \pm 9.08 , AA: 35.51 $\pm 9.08 ; P=0.036$; Figure $1 \mathrm{~F}$ ). In addition, rs8040868 was also related to the LAA\% $(P=0.054)$ and $\mathrm{EV}(P=0.074)$, with a correlation approaching statistical significance. We also assessed the association of these variants with AWT grade because the AWT affects mortality with increasing degrees of emphysema. ${ }^{3}$ The GLM revealed that the rs76071148 and rs8040868 variants were significantly associated with AWT grade $(P=0.046$ and 0.002 , respectively) in spirometry-diagnosed non-COPD subjects.

As associations of the above variants with CT data differed between spirometry-diagnosed non-COPD and COPD individuals, we aimed to determine whether differences were present in those CT parameters and selected variables between the two groups. The results are presented in Table 4. Although the MLD, EV, LAA\%, EI, and AWT significantly differed between the two groups $(P<0.05$ for all), the AWT/AD $(P=0.375)$ and AWT grade $(P=0.625)$ 


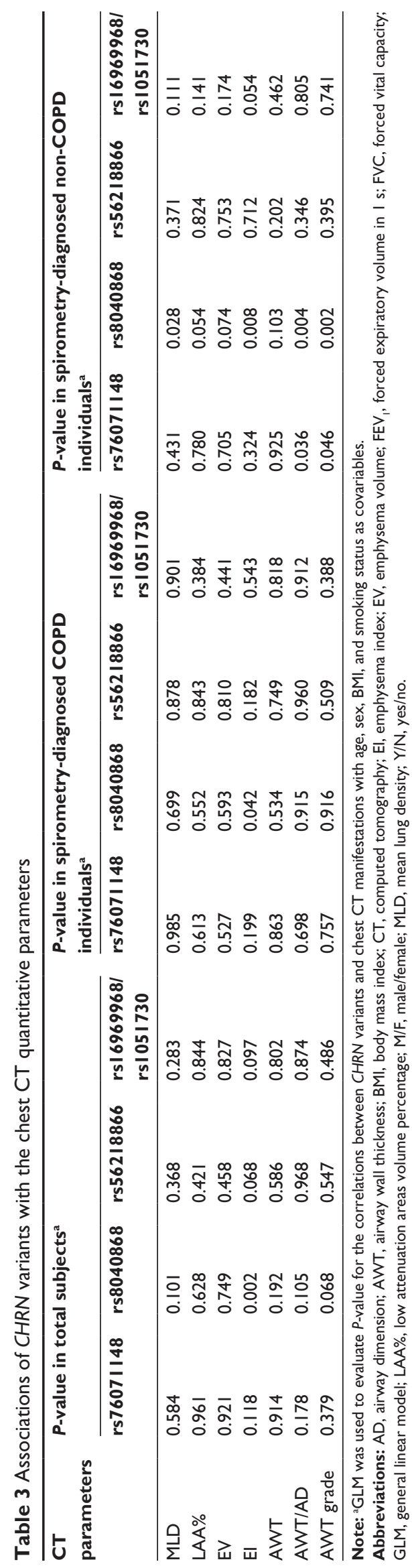

did not significantly differ between the two variants in spirometry-diagnosed non-COPD cases, excluding the possibility of different associations generated by numerical differences. Interestingly, we observed significantly more females, fewer smokers and a higher BMI among subjects in the spirometry-diagnosed non-COPD group than in the COPD group, suggesting a possible "gene-environment" interaction that affected those $\mathrm{CT}$ parameters.

We then analyzed the possible "gene-gene" or "geneenvironment" interaction effects on the above quantitative CT parameters using the GMDR with age as a covariable. We found a significant interaction between the rs 8040868 variant and smoking on the EI $(P=0.010)$ in all subjects. In addition, the rs76071148 and rs8040868 variants and smoking interacted with each other, affecting the AWT/AD $(P=0.011)$ and AWT grade $(P=0.045)$ in spirometry-diagnosed nonCOPD subjects. However, no other significant interaction was observed.

\section{Discussion}

Our knowledge about the role of genetic variants in emphysema and phenotypes of chest CT manifestations remains sorely lacking. In this study, based on a cross-sectional study with a total of 344 emphysema patients with available chest CT data, we analyzed the associations of five genetic variants of a well-known susceptibility gene family for COPD, CHRN, with emphysema phenotypes. We identified two novel associations between the rs76071148 variant and the severity of emphysema as well as an association between rs8040868 and the EI. Interestingly, the stratification analysis grouped by spirometry-diagnosed COPD status revealed that the two variants specially affected AWT/AD and AWT grade in spirometry-diagnosed non-COPD subjects but not in COPD subjects. To the best of our knowledge, this is the first study to investigate the association between CHRN variants and emphysema severity as well as chest CT data in Chinese patients.

To date, multiple association studies, especially genomewide association studies (GWAS), have discovered several genetic loci for emphysema and airway quantitative imaging phenotypes in different ethnicities..$^{17,22,23}$ Accordantly, these previously reported loci for emphysema are also associated with COPD. In a GWAS conducted in non-Hispanic whites and African Americans, Boueiz et al verified the two previously reported associations of COPD susceptibility loci located at $15 \mathrm{q} 25$ near CHRNA5 with the distribution of emphysema at the genome-wide level. ${ }^{17}$ Another GWAS conducted in non-Hispanic whites also revealed that the COPD 
A

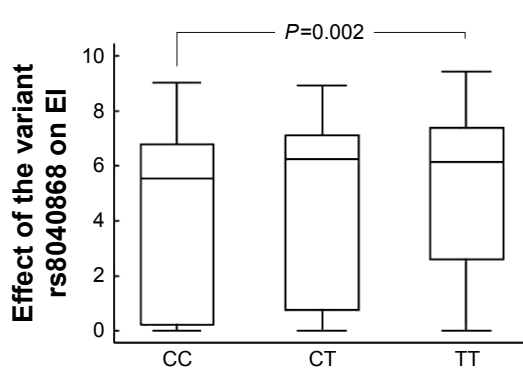

D

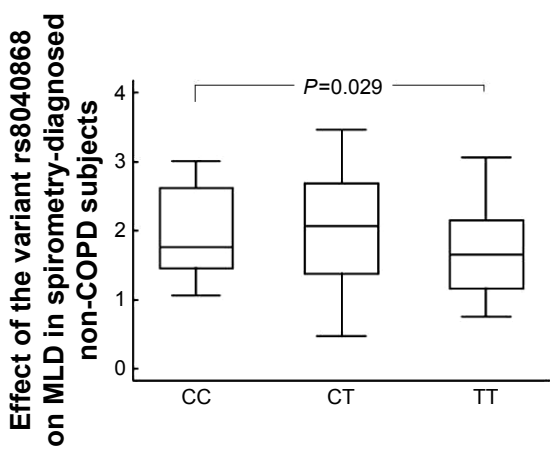

B

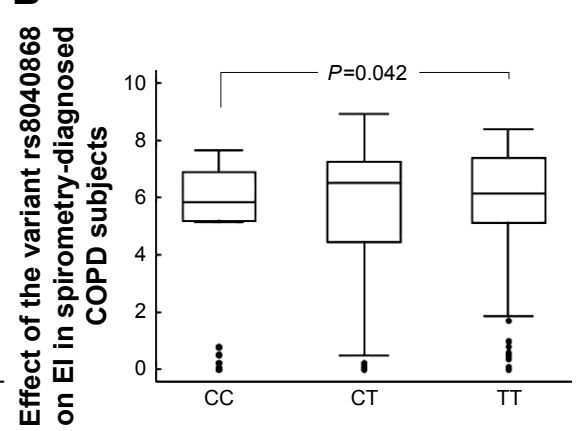

E

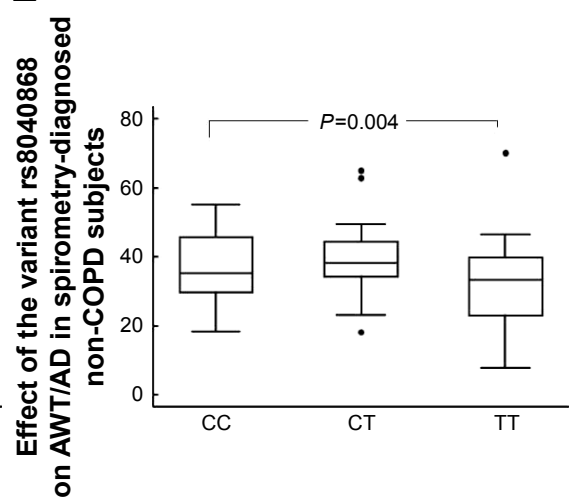

C

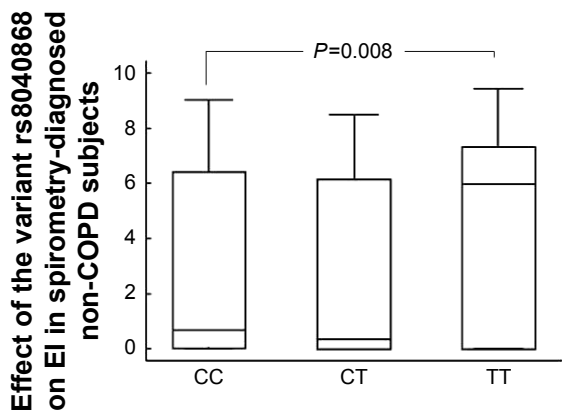

$\mathbf{F}$

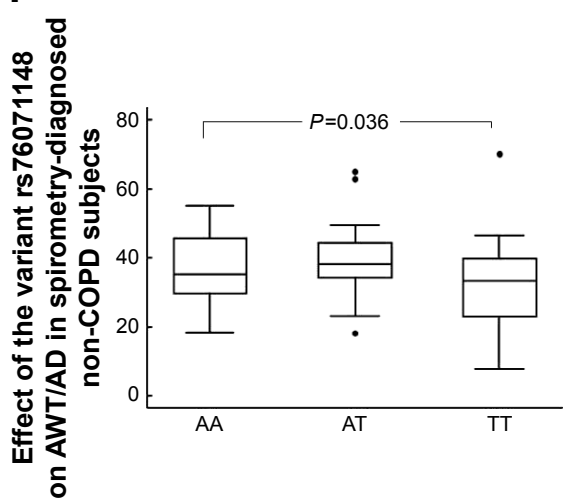

Figure I Effect of the CHRN variants on chest CT manifestations.

Notes: The effect of rs8040868 on El in total samples (A), in spirometry-diagnosed COPD subjects (B) and in spirometry-diagnosed non-COPD subjects (C); (D) the effect of rs8040868 on MLD in spirometry-diagnosed non-COPD subjects; (E) the effect of rs8040868 on AWT/AD in spirometry-diagnosed non-COPD subjects; (F) the effect of rs7607II 48 on AWT/AD in spirometry diagnosed non-COPD subjects. Columns, mean; bars, SD; the one-way ANOVA test was used to assess the differences in above lung functional traits between different genotypes.

Abbreviations: ANOVA, analysis of variance; AWT/AD, airway wall thickness to airway dimensions; CT, computed tomography; COPD, chronic obstructive pulmonary disease; MLD, mean lung density; SD, standard deviation.

Table 4 Distributions of the select characteristics and chest CT parameters with regard to spirometry-diagnosed COPD status

\begin{tabular}{|c|c|c|c|}
\hline Variables & $\begin{array}{l}\text { Spirometry- } \\
\text { diagnosed COPD }\end{array}$ & $\begin{array}{l}\text { Spirometry- } \\
\text { diagnosed } \\
\text { non-COPD }^{a}\end{array}$ & $P$-value ${ }^{b}$ \\
\hline Total no & 241 & 103 & \\
\hline $\operatorname{Sex}(n)$ & $M / F(208 / 33)$ & $M / F(63 / 40)$ & $<0.001$ \\
\hline Age (years) & $70.0 I \pm 9.4 I(24 I)$ & $68.81 \pm 10.84(103)$ & 0.283 \\
\hline BMI $\left(k g / m^{2}\right)$ & $21.66 \pm 3.40(238)$ & $23.43 \pm 3.96(103)$ & $<0.001$ \\
\hline Smoke (n) & $\mathrm{Y} / \mathrm{N}(2 \mathrm{I} 0 / 3 \mathrm{I})$ & $\mathrm{Y} / \mathrm{N}(76 / 27)$ & 0.003 \\
\hline $\operatorname{MLD}\left(\mathrm{g} / \mathrm{cm}^{3}\right)$ & $0.15 \pm 0.06(240)$ & $0.19 \pm 0.07(102)$ & $<0.001$ \\
\hline $\mathrm{EV}\left(\mathrm{g} / \mathrm{cm}^{3}\right)$ & $\mathrm{I}, 619.7 \pm \mathrm{I}, 493.4(240)$ & $703.3 \pm I, 050.4(103)$ & $<0.001$ \\
\hline LAA\% & $30.77 \pm 24.48(240)$ & $14.46 \pm 19.60(103)$ & $<0.001$ \\
\hline $\mathrm{El}$ & $5.39 \pm 2.58(240)$ & $3.26 \pm 3.43(103)$ & $<0.001$ \\
\hline AWT (mm) & $|.22 \pm 0.5|(2 \mid 2)$ & $1.37 \pm 0.42(89)$ & 0.012 \\
\hline AWT/AD & $34.08 \pm 12.02(200)$ & $35.4 \mathrm{I} \pm \mathrm{II} .17$ (88) & 0.375 \\
\hline AWT grade & $0 / 1 / 2(66 / / 24 / 5)$ & $0 / 1 / 2(24 / 59 / 5)$ & 0.625 \\
\hline Post-FEV , (L) & $1.26 \pm 0.62(24 I)$ & $2.01 \pm 0.74(103)$ & $<0.001$ \\
\hline Post-FVC (L) & $2.34 \pm 0.9 I(24 I)$ & $2.57 \pm 0.95(103)$ & 0.038 \\
\hline Post-FEV,/FVC & $0.54 \pm 0.1 I(24 I)$ & $0.79 \pm 0.06(103)$ & $<0.001$ \\
\hline
\end{tabular}

Notes: ${ }^{A} \mathrm{~A}$ small portion of data was missing for certain parameters. ${ }^{\circ}$ The Student's $t$-test and $\chi^{2}$ test were used to assess the $P$-value. Data presented as mean \pm standard deviation unless otherwise specified.

Abbreviations: $A D$, airway dimension; $A W T$, airway wall thickness; $B M I$, body mass index; COPD, chronic obstructive pulmonary disease; CT, computed tomography; El, emphysema index; $\mathrm{EV}$, emphysema volume; $\mathrm{FEV}_{1}$, forced expiratory volume in one second; FVC, forced vital capacity; LAA\%, low attenuation areas volume percentage; $M / F$, male/female; $M L D$, mean lung density; $Y / N$, yes/no. loci at $15 \mathrm{q} 25$ exhibited a genome-wide significant association with visual features of chest CT scans. ${ }^{22}$ Unlike these studies, our study focused on the severity of emphysema, and we consistently demonstrated that the $15 \mathrm{q} 25$ variants for the susceptibility of COPD were nominally associated with the degree of emphysema and the EI. The rs 76071148 variant was found to be related to the severity of emphysema, whereas the rs 8040868 variant was revealed to be correlated with the EI. The rs8040868 variant may also affect the LAA\% and EV. Remarkably, both variants were highly correlated with the AWT/AD and AWT grade in spirometry-diagnosed non-COPD subjects, but not in COPD subjects. The "geneenvironment" interaction may explain the above differences between the two groups because the two variants can significantly interact with smoking in affecting the AWT/AD and AWT grade; furthermore, smoking status significantly differed between the spirometry-diagnosed non-COPD and COPD subjects. However, considering the cross-sectional design of this study with unmatched controls, which could easily produce selection and confounding bias, these findings may have resulted from chance. The study sample size was also small, especially in spirometry-diagnosed 
non-COPD subjects. Furthermore, none of the significant findings reached the significance level in multiple comparison analysis, which also suggests the possibility that our findings were achieved by chance. Nonetheless, the findings of this exploratory study will be of great importance in revealing genetic variants associated with emphysema and the phenotypes of chest CT manifestations.

Although several genetic variants in CHRNA3/5, such as rs1051730 and rs16969968, have been well established to be associated with emphysema, COPD and lung cancer, our findings regarding the rs76071148 variant have not been previously reported..$^{10,17,24,25}$ The rs 8040868 variant was reported to have a significant effect on nicotine dependence in one study. ${ }^{26}$ However, its association with lung cancer risk is controversial. ${ }^{27,28}$ The rs 76071148 variant is a missense variant that causes an amino acid change from histidine to glutamine at codon 462 of CHRNA5. Using SMART software (http://smart. embl-heidelberg.de/), we found that this histidine to glutamine substitution creates novel domains for CHRNA5, suggesting the biological plausibility of the variant. The rs8040868 variant consists of a synonymous substitution at codon 53 of CHRNA3. Functional prediction of this variant using the SNP info site (http://snpinfo.niehs.nih.gov/) demonstrated that this variant is located at the exonic splicing site, which may affect the process of alternative splicing in CHRNA3 and lead to abnormal CHRNA3 expression or isoforms. ${ }^{29}$ Consistently, the "genotype-eQLT phenotype" correlation with the GTEx database (http://www.gtexportal.org/home/ eqtls/) indicated a significant correlation between rs 8040868 and CHRNA3 expression in muscle tissues. ${ }^{30}$ The variant was also significantly correlated with CHRNA5 expression in various samples. Collectively, this evidence indicates that the two variants are functional and contribute to the severity of emphysema as well as CT imaging manifestations.

We failed to detect a clear association between rs 1051730 and rs16969968 and emphysema severity or quantitative chest CT parameters. Although the two variants have been demonstrated in several well-known GWAS to represent susceptible loci for emphysema, COPD and lung cancer in Caucasians, we did not observe a significant association between these variants and emphysema. ${ }^{10,17,24,25}$ This may be due to their rare frequency in Chinese patients, whereas they are common in Caucasian populations. Moreover, we failed to test an association between these variants and body plethysmography or diffusing capacity of the lung for carbon monoxide data because we lacked sufficient data.

The differences in individual characteristics and chest CT manifestations between the spirometry-diagnosed non-COPD and COPD subjects are meaningful because all patients were randomly recruited. The former are more likely to be females, non-smokers and to have a higher BMI and superior CT manifestations than the latter. Therefore, the spirometry-diagnosed non-COPD group is likely to be ignored in the diagnosis. As the rs76071148 and rs8040868 variants are likely to affect emphysema in spirometry-diagnosed non-COPD subjects, they represent promising biomarkers to evaluate emphysema risk in this allelomorphic population.

\section{Conclusion}

Overall, the conclusion of our study is that the rs 76071148 variant of CHRNA5 and the rs 8040868 variant of CHRNA3 are associated with the severity of emphysema and the EI, respectively. Furthermore, the effects of these factors on chest $\mathrm{CT}$ manifestations are unique in spirometry-diagnosed non-COPD subjects, suggesting their role as genetic predictors of emphysema risk. Further validation in other populations is, therefore, warranted.

\section{Acknowledgments}

The authors thank everyone who participated in this study and its funders. In particular, they also thank Hua He (The First Affiliated Municipal Hospital, Guangzhou Medical University) and Wei Hong (The State Key Laboratory of Respiratory Diseases, The First Affiliated Hospital, Guangzhou Medical University) for their help with monitoring, data collection, and conducting the statistical analysis.

This work was supported by the National Natural Science Foundation of China (81170043) and the Science and Technology Project of Guangdong (2013B021800069). The funders had no role in the study design, data collection and analysis, decision to publish, or preparation of the manuscript.

\section{Disclosure}

The authors report no conflicts of interest in this work.

\section{References}

1. Tho NV, Ryujin Y, Ogawa E, et al. Relative contributions of emphysema and airway remodelling to airflow limitation in COPD: consistent results from two cohorts. Respirology. 2015;20(4):594-601.

2. Lutchmedial SM, Creed WG, Moore AJ, et al. How common is airflow limitation in patients with emphysema on CT scan of the chest? Chest 2015;148(1):176-184.

3. Johannessen A, Skorge TD, Bottai M, et al. Mortality by level of emphysema and airway wall thickness. Am J Respir Crit Care Med. 2013 187(6):602-608.

4. McCloskey SC, Patel BD, Hinchliffe SJ, et al. Siblings of patients with severe chronic obstructive pulmonary disease have a significant risk of airflow obstruction. Am J Respir Crit Care Med. 2001;164(8 Pt 1) 1419-1424.

5. Yang L, Yang X, Ji W, et al. Effects of a functional variant c.353T $>$ C in snail on risk of two contextual diseases. Chronic obstructive pulmonary disease and lung cancer. Am J Respir Crit Care Med. 2014; 189(2):139-148. 
6. Yang L, Lu X, Qiu F, et al. Duplicated copy of CHRNA7 increases risk and worsens prognosis of COPD and lung cancer. Eur J Hum Genet. 2015;23(8):1019-1024.

7. Hobbs BD, Parker MM, Chen H, et al. Exome array analysis identifies a common variant in IL27 associated with chronic obstructive pulmonary disease. Am J Respir Crit Care Med. 2016;194(1):48-57.

8. Horita N, Miyazawa N, Tomaru K, et al. Vitamin D binding protein genotype variants and risk of chronic obstructive pulmonary disease: a meta-analysis. Respirology. 2015;20(2):219-225.

9. Zhang J, Summah H, Zhu YG, et al. Nicotinic acetylcholine receptor variants associated with susceptibility to chronic obstructive pulmonary disease: a meta-analysis. Respir Res. 2011;12:158.

10. Pillai SG, Ge D, Zhu G, et al. A genome-wide association study in chronic obstructive pulmonary disease (COPD): identification of two major susceptibility loci. PLoS Genet. 2009;5(3):e1000421.

11. Wilk JB, Shrine NR, Loehr LR, et al. Genome-wide association studies identify CHRNA5/3 and HTR4 in the development of airflow obstruction. Am J Respir Crit Care Med. 2012;186(7):622-632.

12. Lee JH, Cho MH, Hersh CP, et al. Genetic susceptibility for chronic bronchitis in chronic obstructive pulmonary disease. Respir Res. 2014; $15: 113$.

13. Zhao Z, Peng F, Zhou Y, et al. Exon sequencing identifies a novel CHRNA3-CHRNA5-CHRNB4 variant that increases the risk for chronic obstructive pulmonary disease. Respirology. 2015;20(5):790-798.

14. Zhao Z, Zhou Y, Li Y, et al. An efficient method to genotype the polymorphisms of cholinergic nicotinic receptor subunit genes and their associations with COPD onset risk. Exp Lung Res. 2016;42(5): 267-274.

15. Lambrechts D, Buysschaert I, Zanen P, et al. The $15 q 24 / 25$ susceptibility variant for lung cancer and chronic obstructive pulmonary disease is associated with emphysema. Am J Respir Crit Care Med. 2010;181(5): 486-493.

16. Castaldi PJ, Cho MH, San Jose Estepar R, et al. Genome-wide association identifies regulatory Loci associated with distinct local histogram emphysema patterns. Am J Respir Crit Care Med. 2014;190:399-409.

17. Boueiz A, Lutz SM, Cho MH, et al. Genome-wide association study of the genetic determinants of emphysema distribution. Am J Respir Crit Care Med. 2017;195(6):757-771.

18. Pillai SG, Kong X, Edwards LD, et al. Loci identified by genome-wide association studies influence different disease-related phenotypes in chronic obstructive pulmonary disease. Am J Respir Crit Care Med. 2010;182(12):1498-1505.
19. Miller MR, Hankinson J, Brusasco V, et al. Standardisation of spirometry. Eur Respir J. 2005;26:319-338.

20. Kim SS, Seo JB, Lee HY, et al. Chronic obstructive pulmonary disease: lobe-based visual assessment of volumetric CT by Using standard images - comparison with quantitative $\mathrm{CT}$ and pulmonary function test in the COPD Gene study. Radiology. 2013;266(2):626-635.

21. Lou XY, Chen GB, Yan L, et al. A generalized combinatorial approach for detecting gene-by-gene and gene-by-environment interactions with application to nicotine dependence. Am J Hum Genet. 2007; 80(6):1125-1137.

22. Halper-Stromberg E, Cho MH, Wilson C, et al. Visual assessment of chest $\mathrm{CT}$ images is independently useful for genetic association analysis in studies of chronic obstructive pulmonary disease. Ann Am Thorac Soc. 2017;14(1):33-40.

23. Cho MH, Castaldi PJ, Hersh CP, et al. A genome-wide association study of emphysema and airway quantitative imaging phenotypes. $\mathrm{Am}$ J Respir Crit Care Med. 2015;192(5):559-569.

24. Cho MH, McDonald ML, Zhou X, et al. Risk loci for chronic obstructive pulmonary disease: a genome-wide association study and meta-analysis. Lancet Respir Med. 2014;2(3):214-225.

25. Hung RJ, McKay JD, Gaborieau V, et al. A susceptibility locus for lung cancer maps to nicotinic acetylcholine receptor subunit genes on 15q25. Nature. 2008;452(7187):633-637.

26. Li MD, Xu Q, Lou XY, et al. Association and interaction analysis of variants in CHRNA5/CHRNA3/CHRNB4 gene cluster with nicotine dependence in African and European Americans. Am J Med Genet B Neuropsychiatr Genet. 2010;153B(3):745-756.

27. Chikova A, Bernard HU, Shchepotin IB, et al. New associations of the genetic polymorphisms in nicotinic receptor genes with the risk of lung cancer. Life Sci. 2012;91(21-22):1103-1108.

28. Lou G, Zhang Y, Bao W, et al. Association between polymorphisms in CHRNA3 and PHACTR2 gene and environment and NSCLC risk in Chinese population. Acta Biochim Pol. 2014;61(4):765-768.

29. Xu Z, Taylor JA. SNP info: integrating GWAS and candidate gene information into functional SNP selection for genetic association studies. Nucleic Acids Res. 2009;37:W600-W605.

30. Consortium GT. Human genomics. The Genotype-Tissue Expression (GTEx) pilot analysis: multitissue gene regulation in humans. Science. 2015;348(6235):648-660. 


\section{Supplementary material}

Table SI Distributions of CHRN variants and their associations with degree of emphysema stratified by spirometry-diagnosed COPD status

\begin{tabular}{|c|c|c|c|c|c|}
\hline \multirow[t]{2}{*}{ Variants } & \multicolumn{3}{|c|}{ Degree of emphysema } & \multirow[t]{2}{*}{$P$-value ${ }^{a}$} & \multirow[t]{2}{*}{ OR $(95 \% \mathrm{Cl})^{\mathrm{b}}$} \\
\hline & Mild n (\%) & Moderate n (\%) & Severe n (\%) & & \\
\hline Spirometry-diagnosed COPD individuals & 58 & 70 & 113 & & \\
\hline rs7607II48 & & & & 0.068 & \\
\hline TT & $32(55.2)$ & $42(60.0)$ & $64(56.6)$ & & 1.00 (Ref) \\
\hline AT & $19(32.7)$ & $24(34.3)$ & $47(41.6)$ & & $0.67(0.39-1.16)$ \\
\hline AA & $7(12.1)$ & $4(5.7)$ & $2(1.8)$ & & $2.53(0.8 I-7.93)$ \\
\hline AT vs TT + AA & & & & & $0.61(0.36-1.04)$ \\
\hline rs8040868 & & & & 0.031 & \\
\hline TT & $24(4 \mid .4)$ & $26(37.1)$ & $45(39.8)$ & & 1.00 (Ref) \\
\hline CT & $21(36.2)$ & $37(52.9)$ & $60(53.1)$ & & $0.76(0.44-1.31)$ \\
\hline $\mathrm{CC}$ & $13(22.4)$ & $7(10.0)$ & $8(7.1)$ & & $1.89(0.82-4.35)$ \\
\hline rs56218866 & & & & 0.360 & \\
\hline TT & $45(77.6)$ & $48(68.6)$ & $87(77.0)$ & & I.00 (Ref) \\
\hline CT & $12(20.7)$ & $20(28.6)$ & $26(23.0)$ & & $0.98(0.54-1.77)$ \\
\hline $\mathrm{CC}$ & $\mathrm{I}(\mathrm{l} .7)$ & $2(2.8)$ & $0(0.0)$ & & $2.74(0.32-23.6)$ \\
\hline rs16969968/rs105I730 & & & & 0.678 & \\
\hline GG & $55(94.8)$ & $64(91.4)$ & $103(91.1)$ & & 1.00 (Ref) \\
\hline AG & $3(5.2)$ & $6(8.6)$ & $10(8.9)$ & & $0.69(0.27-1.78)$ \\
\hline Spirometry-diagnosed non-COPD individuals & 10 & 16 & 77 & & \\
\hline rs7607।I48 & & & & 0.439 & \\
\hline $\mathrm{TT}$ & $6(60.0)$ & II (68.8) & $37(48.0)$ & & 1.00 (Ref) \\
\hline AT & $4(40.0)$ & $5(3 \mid .2)$ & $34(44.2)$ & & $0.55(0.2 \mathrm{I}-\mathrm{I} .45)$ \\
\hline AA & $0(0.0)$ & $0(0.0)$ & $6(7.8)$ & & - \\
\hline AT vs TT + AA & & & & & $0.61(0.23-1.60)$ \\
\hline rs8040868 & & & & 0.167 & \\
\hline $\mathrm{TT}$ & $6(60.0)$ & $10(62.6)$ & $29(37.7)$ & & 1.00 (Ref) \\
\hline $\mathrm{CT}$ & $4(40.0)$ & $3(18.7)$ & $33(42.8)$ & & $0.43(0.15-1.23)$ \\
\hline $\mathrm{CC}$ & $0(0.0)$ & $3(18.7)$ & $15(19.5)$ & & $0.36(0.08-1.56)$ \\
\hline $\mathrm{CT}+\mathrm{CC}$ vs TT & & & & & $0.4 I(0.16-1.05)$ \\
\hline rs562I8866 & & & & 0.520 & \\
\hline $\mathrm{TT}$ & $8(80.0)$ & 15 (93.7) & $64(83.1)$ & & I.00 (Ref) \\
\hline $\mathrm{CT}$ & $2(20.0)$ & I (6.3) & $13(16.9)$ & & $0.93(0.23-3.72)$ \\
\hline $\mathrm{CC}$ & $0(0.0)$ & $0(0.0)$ & $0(0.0)$ & & - \\
\hline rsI6969968/rsI05I730 & & & & 0.049 & \\
\hline GG & $10(100.0)$ & $13(81.3)$ & $74(96.1)$ & & I.00 (Ref) \\
\hline AG & $0(0.0)$ & $3(18.7)$ & $3(3.9)$ & & $2.13(0.37-12.3)$ \\
\hline
\end{tabular}

Notes: ${ }^{a}$ The two-sided $\chi^{2}$ test between difference groups. ${ }^{b}$ Adjusted in a logistic regression model with age, sex, smoking status and BMI as covariables.

Abbreviations: BMI, body mass index; $\mathrm{Cl}$, confidence interval; COPD, chronic obstructive pulmonary disease; OR, odds ratio; Ref, reference.

\section{Publish your work in this journal}

The International Journal of COPD is an international, peer-reviewed journal of therapeutics and pharmacology focusing on concise rapid reporting of clinical studies and reviews in COPD. Special focus is given to the pathophysiological processes underlying the disease, intervention programs, patient focused education, and self management protocols.

\section{Dovepress}

This journal is indexed on PubMed Central, MedLine and CAS. The manuscript management system is completely online and includes a very quick and fair peer-review system, which is all easy to use. Visit http://www.dovepress.com/testimonials.php to read real quotes from published authors. 